\title{
ESTABILIDADE DE AGREGADOS DE UM PLANOSSOLO SOB DIFERENTES SISTEMAS DE MANEJ $0^{(1)}$
}

\author{
C. L. R. LIMA(2), E. A. PAULETTO(3), A. S. $\operatorname{GOMES}^{(4)}$ \& J . B. SILVA(5)
}

\begin{abstract}
RESUMO
Os solos de várzea no Rio Grande do Sul, considerando a heterogeneidade do material de origem e os diferentes graus de hidromorfismo, apresentam grandes variações nas suas características morfológicas, físicas, químicas e mineralógicas. Sua principal exploração tem ocorrido por meio do binômio: arroz irrigado e pecuária de corte, freqüentemente de baixa rentabilidade. A introdução de espécies de sequei ro em rotação e/ou sucessão com o arroz irrigado vem sendo apregoada para aumentar a utilização desses solos, permitir maior controle de plantas daninhas do arroz irrigado e melhorar o seu estado físico degradado mediante sistemas que envolvam menor mobilização do solo. Nesse contexto, avaliou-se o efeito de sistemas de preparo convencional (SC), cultivo mínimo (CM), semeadura direta (SD) e pré-germinado (PG) sobre o estado de agregação de um Planossolo Hidromórfico eutrófico, por meio dos seguintes atributos: distribuição de agregados estáveis em água em diferentes classes de tamanho e diâmetro médio ponderado dos agregados (DMP). O experimento vem sendo realizado desde 1995/96 e encontra-se instalado na Estação Experimental de Terras Baixas (ETB) da Embrapa - Clima Temperado, no município do Capão do Leão (RS). Após três anos, o sistema de manejo SD favoreceu a formação de agregados de maior tamanho, originando maior diâmetro médio ponderado de agregados, enquanto o sistema de plantio PG proporcionou a maior concentração de agregados do solo na classe de menor tamanho, com menor diâmetro médio ponderado dos agregados. 0 diâmetro médio ponderado de agregados do solo correlacionou-se linear e positivamente com o C-orgânico, não apresentando correlação com a argila dispersa em água.
\end{abstract}

Termos de indexação: sistema de cultivo, agregação, solos de terras baixas.

\footnotetext{
(1) Parte da Tese de Mestrado do primeiro autor, apresentada à Faculdade de Agronomia Eliseu Maciel, curso de Pós-Graduação em Agronomia, Universidade Federal de Pelotas - UF Pel. Recebido para publicação em dezembro de 2001 e aprovado em novembro de 2002.

(2) Pós-Graduanda em Agronomia, Universidade Federal de Pelotas - UFPel. Caixa Postal 354, CEP 96010-900 Pelotas (RS). E-mail: clrlima@esalq.usp.br

(3) Professor do Departamento de Solos da Faculdade de Agronomia Eliseu Maciel, UFPel. E-mail: pauletto@ufpel.tche.br

(4) Pesquisador do Centro de Pesquisa Agropecuária de Clima Temperado - CPACT/E MBRAPA. Caixa Postal 403, CEP 96001- 970 Pelotas (RS). E-mail: algenor@cpact.embrapa.br

(5) Professor do Instituto de Física e Matemática, UFPel. E-mail: jbsilva@ufpel.tche.br
} 


\title{
SUMMARY: AGGREGATE STABILITY OF AN ALBAQUALF UNDER DIFFERENT TILLAGE SYSTEMS
}

\begin{abstract}
The wetland soils in the state of Rio Grande do Sul present wide variation in their morphol ogical, physical, chemical and mineralogical characteristics duetotheheterogeneity of the parent material and different degrees of hydromorphism. Sincetheir main uses for flooded rice and cattl e breeding are frequently low-profit activities, theintroduction of dry land crop species in rotation and/ or succession with flooded rice has been called for. By broadening the spectrum of wetland soil use, a better weed control for flooded rice and an enhanced physical state of the degraded soil by management systems of reduced soil disturbance would become possible In this context, the effect of tillage systems on the aggregation state of an Albaqualf has been eval uated: conventional tillage(CT), mi nimum tillage (MT), notillage (NT) and pregerminated (PG), by means of thefollowing attributes: distribution of stable water aggregates in different size classes and mean weight diameter (MWD) of theaggregates. Theexperiment has ben conducted since 1995/ 96 and is installed in the Estação Experimental de Terras Baixas (Experimental Station Lowlands-ESL) of Embrapa (Brazilian agricultural research organization), Clima Temperado, district of Capão do Leão (RS). After thre years, the NT system favored an aggregate formation of larger sizes, originating largest MWD, while the system PG pushed the largest aggregate concentration of thesoil into thesmal lest sizeclass, with thesmallest aggregateMWD. The MWD was lineal and positively correlated with theorganic C, not presenting any correlation with the clay dispersed in water.
\end{abstract}

Index terms: tillage system, aggregation, lowland soil.

\section{INTRODUÇÃO}

A qualidade estrutural do sol otem sido associada às condições físicas favoráveis à emergência de plântulas, desenvolvimento radicular, aeração, infiltração e movimento de água no perfil do solo. Doran \& Parkin (1994) definem qualidade de sol o como sendo a capacidade do sol o de exercer várias funções, dentro dos limites do uso da terra e do ecossistema, com vistas em sustentar a produtividade biológica, manter ou melhorar a qualidade ambiental e contribuir para a saúde das plantas, dos animais e dos homens.

Segundo Eash et al. (1994), a formação da estrutura do solo resulta na formação dos agregados do solo. Aspectos relacionados com a estrutura podem ser quantitativamente caracterizados pela distribuição de tamanho deagregados estáveis em água (Arshad et al., 1996).

A formação ea estabilização de agregados ocorrem simultaneamente mediante a atuação de processos físicos, químicos e biológicos no solo. Bayer \& Mielniczuk (1999) destacam que a formação de agregados é influenciada não só pelas forças físicas envolvidas no umedecimento e secamento, congel amento e descongelamento, mas também pela compressão das raízes e que, após a aproximação das partículas minerais, o C- orgânico é de importância fundamental na estabilização dos agregados.

Os compostos orgânicos participam das ligações entreas partículas individuais do solo, atuando como agentes cimentantes das unidades estruturais pelas suas características desuperfície, as quais controlam diversas reações importantes no solo e contribuem para determinar seu comportamento frente às práticas de manejo agrícola eal terações noambiente. A participação destes compostos pode influir ainda na capacidade produtiva do sol o por mei o de diversos processos, tais como: amenização do efeito de elementos e compostos tóxicos, capacidade de infiltração e retenção de água, aeração, atividade biológica, capacidade de troca de cátions e disponibilidade de nutrientes (Silva et al., 2000a).

A agregação do solo é influenciada, ainda, pela composição textural (Castro Filho et al., 1998), condições cl imáticas, ação de microrganismos, raízes de plantas, resíduos vegetais e pelo próprio tracionamento do solo (Silva \& Miel niczuk, 1997b).

A estabilidade dos agregados também pode ser alterada por diferentes sistemas de manejo. Segundo Eltz et al. (1989), o sistema de manejo semeadura direta proporciona maior tamanho de agregados estáveis em água, quando comparado ao preparo convencional, possivelmente pela nãodestruição mecânica dos agregados causada pelos implementos de preparo e pel a proteção quea pal ha ofereceà superfície do sol o. Carpenedo \& Miel niczuk (1990) concordam com a eficiência da semeadura direta na melhoria da estabilidade dos agregados do solo em relação ao preparo convencional .

Tratando mais especificamente de solos de várzea, manejados com monocultivo e em preparo 
convencional do solo, vêm-se buscando alternativas que reduzam a infestação de plantas daninhas e mel horem o estado físico do sol o. O objetivo disto é viabilizar a sua utilização de forma mais intensa com arroz irrigado e, ou, com culturas de sequeiro, visando ao aumento de produtividade e à menor agressão ao meio ambiente.

Considerando que sistemas de manejo diferenciados alteram a estrutura do solo, objetivou-se avaliar o estado de agregação de um Planossolo sob diferentes sistemas de manejo em um experimento com duração de três anos, por meio da distribuição deagregados estáveis em água em diferentes classes e diâmetro médio ponderado (DMP).

\section{MATERIAL E MÉTODOS}

O presente trabalho foi realizado sobre um experimento de campo situado no município de Capão do Leão, durante três safras agrícolas consecutivas, a partir de 1995/96, na Estação Experimental deTerras Baixas da Embrapa Clima Temperado, RS. Utilizou-se um Planossolo Hidromórfico eutrófico solódico textura média/ argilosa, de acordo com EMBRAPA (1999), pertencente à Unidade de Mapeamento Pelotas (Brasil, 1973) e, segundo o Soil Survey Staff (1990), classificado como um Albaqualf.

O delineamento experimental adotado foi o de blocos ao acaso com quatro repetições. Os sistemas de manejo do solo estudados foram: preparo convencional (SC), cultivo mínimo (CM), semeadura direta (SD), além de um tratamento com arroz prégerminado (PG).

No sistema convencional, o sol o foi preparado por meio de uma aração profunda e uma ou duas gradagens, dependendo do nivelamento do solo. O cultivo mínimo foi realizado no final do inverno e início da primavera com uma ou duas passadas de grade, ea semeadura do arroz sob a flora de sucessão normal mente composta por plantas daninhas, como o arroz vermel ho. A semeadura direta consistiu na semeadura do arroz sob a cobertura vegetal do solo, sendo o azevém a espécie de inverno utilizada. O sistema de cultivo pré-germinado, no Rio Grande do Sul, consistiu num preparo do solo semel hante ao convencional realizado em área previamente sistematizada, adicionado de um alisamento superficial na presença de uma lâmina de água, na qual foram colocadas as sementes pré-germinadas, com radicel as com comprimento de 2 a 3 mm (IRGA, 2001).

Nestas áreas, foram coletadas amostras deformadas nas camadas de 0-2,5; 2,5-5,0; 5-10 e 10$20 \mathrm{~cm}$, as quais foram processadas no Laboratório deFísica do Solo da Faculdade deAgronomia Eliseu Maciel. No laboratório, as amostras foram secas à sombra e destorroadas manualmente através de seus planos de fraqueza de maneira suave para não provocar compactação ou ruptura dos agregados e, após, passadas em peneira com abertura de mal ha $9,52 \mathrm{~mm}$. A distribuição de tamanho dos agregados estáveis em água em diferentes classes e o cálculo do diâmetro médio ponderado (DMP) foram feitos com base no método descrito em Palmeira et al. (1999), segundo o princípio estabel ecido por Kemper \& Rosenau (1986), que utiliza o aparel ho de oscilação vertical Yoder (1936). No peneiramento, utilizou-se um jogo de peneiras com abertura de mal ha de 4,76; 2,0; 1,0; 0,25 e 0,105 mm.

Complementarmente, foram determinados oteor de C-orgânico, pelo método de Walkley-Black (Tedesco et al., 1995), e de argila naturalmente dispersa em água, pelo método da pipeta descrito por Gee \& Bauder (1986).

Os resultados obti dos foram submetidos à análise de variância utilizando para comparação de médias otestedeDuncan a $5 \%$, para as variáveis: distribuição percentual de agregados estáveis em água em diferentes classes de tamanho, diâmetro médio ponderado dos agregados, teor deC-orgânico eargila naturalmente dispersa em água. A análise de correlação linear simples foi utilizada para as variáveis: DMP, C-orgânico e argila naturalmente dispersa em água, para cada camada amostrada, utilizando o Sistema de Análise Estatística SANEST (Zonta et al., 1984).

\section{RESULTADOS E DISCUSSÃO}

Valores médios de agregados estáveis em água, em diferentes classes de tamanho, e de C-orgânico, eargila naturalmente dispersa em água (ADA) e de diâmetro médio ponderado (DMP) dos agregados DMP encontram-se na figura 1 e no quadro 1 , respectivamente.

Em termos gerais, as maiores quantidades de agregados na dasse de maior tamanho $(\mathrm{C} 1=9,52 \mathrm{a}$ $4,76 \mathrm{~mm}$ ) foram proporcionadas pel o sistema SD, em todas as camadas, enquanto, na classe de menor tamanho (C6 < 0,105 mm), as maiores quantidades foram condicionadas pelo sistema PG. Esse fato está relacionado com o próprio sistema de preparo que é feito pela formação da lama, que destrói quase que por completo os agregados de maior tamanho, favorecendo, desta forma, a maior quantidade de menores agregados. Segundo Peña et al. (1996), Teixeira (1998), Palmeira et al. (1999) e Silva et al. (2000b), a utilização da SD poderá concorrer para a melhoria da estrutura do solo.

Os resultados obtidos indicam que os sistemas de manejo testados não exerceram efeitos significativos sobre as quantidades de agregados de 1 a 0,105 mm de diâmetro, istoé, nas dasses intermediárias (C4 e C5), em todas as camadas (Figura 1). 

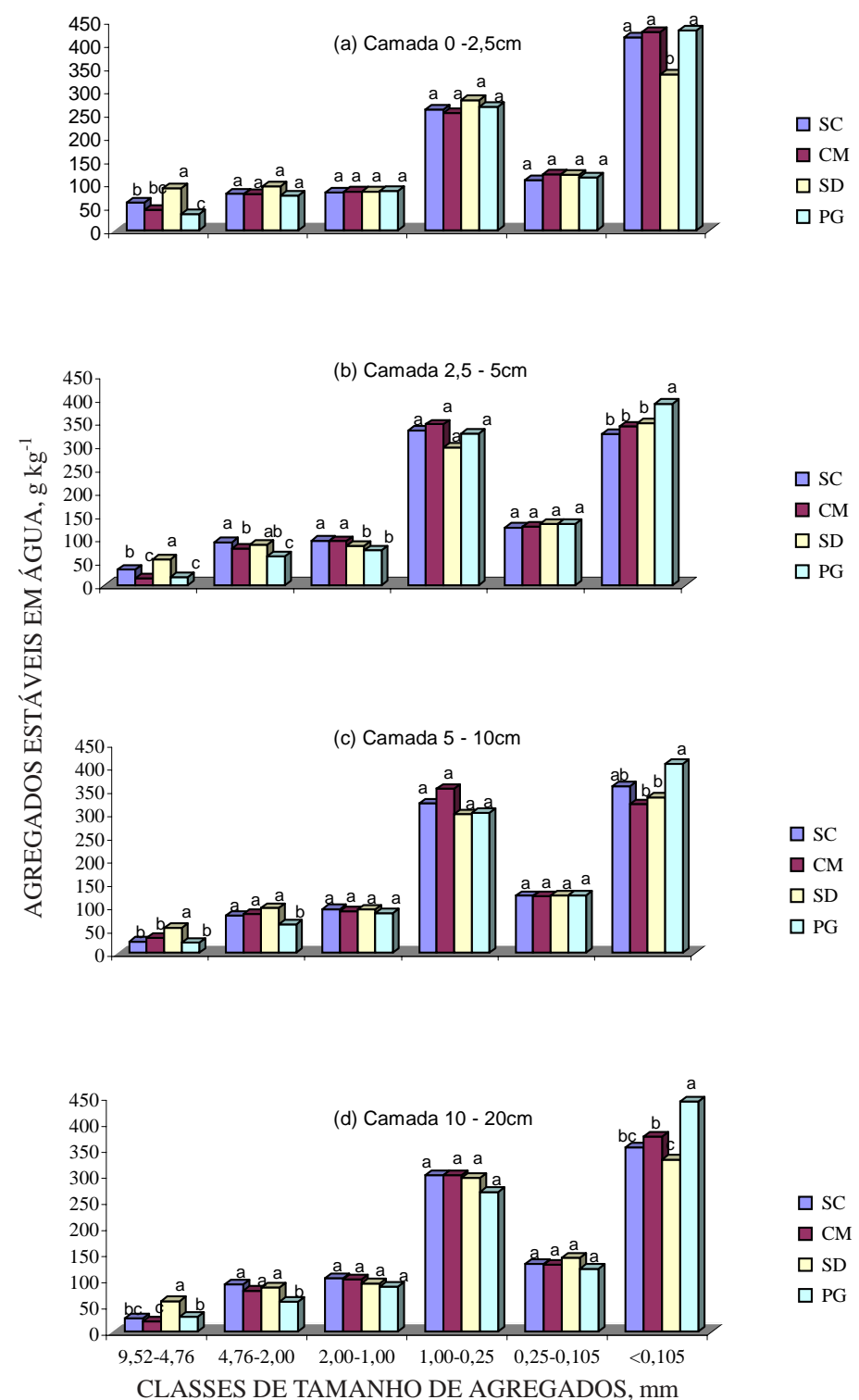

SC: sistema convencional, CM: cultivo mínimo, SD: semeadura direta, PG: pré-germinado.

Figura 1. Valores médios de agregados estáveis em água em diferentes classes de distribuição de tamanho de um Planossolo, considerando sistemas de manejo e camadas. Valores seguidos pela mesma letra dentro de cada classe, não diferem si gnificativamente pelo teste de Duncan a $5 \%$.

Da mesma forma, foi possível constatar um acúmulo de agregados de 1,00 a $0,25 \mathrm{~mm}$ de diâmetro (C4) e menores que 0,105 mm (C6). Palmeira et al. (1999), no mesmo tipo de solo, submetido a diferentes sistemas de manejo por um período de 10 anos, também observaram um acúmulo de agregados nestas classes. Tisdall \& Oades (1982) explicam que o acúmulo de agregados nas classes inferiores a 1,00 mm ocorre por serem eles mais estáveis por ocasião do umedecimento e não sofrerem destruição por práticas agrícolas, visto que são constituídos, predominantemente, por partículas de 2-20 $\mu \mathrm{m}$ de diâmetro, unidas em cadeias por diversas substâncias cimentantes, como materiais orgânicos, óxidos cristalinos e aluminossilicatos desordenados.

A aplicação do teste de Duncan sobre os valores médios das variáveis anal isadas indica que osistema SD proporcionou, em todas as camadas estudadas, os maiores valores de DMP e de C-orgânico (Quadro 1), os quais foram, em termos estatísticos, diferenciados daqueles correspondentes aos dos demais sistemas.

Os maiores teores de C-orgânico proporcionados pelo sistema SD devem ter contribuído para a presença de maior quantidade de agregados nas classes de maior tamanho. 
Bayer \& Mielniczuk (1997) explicam que o maior conteúdo de C-orgânico nas camadas superficiais de solos não revolvidas, comparativamente ao dos demais preparos, é devido à menor taxa de perda de matéria orgânica e à localização superficial do material orgânico adicionado por sistemas decultivo. Para Silva et al. (2000a), sistemas de manejo que empregam operações com intenso revolvimento revelam taxa de perda decarbononormalmentemaior que a taxa de adição, resultando em decréscimo dos teores de matéria orgânica.

Percebeu-se diminuição do DMP dos agregados em profundidade, principalmente em relação à primeira camada, em decorrência, provavelmente, da diminuição dos teores de C-orgânico em profundidade(Quadro 1). Observa-se que o sistema SD apresentou os maiores valores de DMP e o sistema PG os menores, refletindo nos dados apresentados anteriormente com relação à distribuiç̧ão dos agregados nas diferentes classes de tamanho (Figura 1).
Sobre a argila naturalmente dispersa em água (ADA), não foram verificados efeitos significativos dos tratamentos (Quadro 1). Palmeira et al. (1999) também não encontraram diferenças significativas sobreos teores deargila naturalmente dispersa entre os tratamentos estudados.

Percebe-se, pelo quadro 2, que, em todas as camadas do solo estudado, houve correlação altamente significativa entre os teores de C-orgânico e DMP e maior coeficiente de correlação linear na camada de $0-2,5 \mathrm{~cm}$.

Não foram encontradas correlações significativas entre o DMP e a argila naturalmente dispersa em água (ADA) (Quadro 2). Silva \& Miel niczuk (1997a) e Reichert et al. (1993) também não encontraram correlação significativa entre estes dois atributos. Silva \& Mielniczuk (1998) evidenciaram que a argila e o grau de dispersão exerceram efeitos na agregação do solo, porém foram insuficientes para explicar as variações entreos valores de DMP encontrados entre dois diferentes tipos de solos.

Quadro 1. C-orgânico, argila naturalmente dispersa em água (ADA) e diâmetro médio ponderado (DMP) dos agregados de um Planossolo, considerando sistemas de manejo e camadas de solo

\begin{tabular}{|c|c|c|c|c|c|}
\hline \multirow{2}{*}{ Variável } & \multirow{2}{*}{ Camada } & \multicolumn{4}{|c|}{ Sistema de manejo(1) } \\
\hline & & SC & $\mathbf{C M}$ & SD & PG \\
\hline & $\mathrm{cm}$ & & & & \\
\hline C-orgânico (g kg-1) & $\begin{array}{c}0-2,5 \\
2,5-5,0 \\
5,0-10 \\
10-20\end{array}$ & $\begin{array}{c}11,28 b^{(2)} \\
9,44 b \\
9,83 b \\
9,01 b\end{array}$ & $\begin{array}{c}10,58 b c \\
10,00 b \\
9,93 b \\
8,06 c\end{array}$ & $\begin{array}{r}14,68 a \\
11,34 a \\
11,00 a \\
9,96 a\end{array}$ & $\begin{array}{r}10,09 c \\
9,43 b \\
8,60 c \\
8,10 c\end{array}$ \\
\hline ADA $\left(\mathrm{g} \mathrm{kg}^{-1}\right)$ & $\begin{array}{c}0-2,5 \\
2,5-5,0 \\
5,0-10 \\
10-20\end{array}$ & $\begin{array}{l}49,83 a \\
68,90 a \\
77,06 a \\
74,60 a\end{array}$ & $\begin{array}{l}56,11 a \\
74,94 a \\
74,25 a \\
84,05 a\end{array}$ & $\begin{array}{l}57,79 a \\
75,85 a \\
84,33 a \\
87,93 a\end{array}$ & $\begin{array}{l}59,71 a \\
72,29 a \\
76,11 a \\
83,36 a\end{array}$ \\
\hline DMP (\%) & $\begin{array}{c}0-2,5 \\
2,5-5,0 \\
5,0-10 \\
10-20\end{array}$ & $\begin{array}{l}1,01 b \\
0,93 b \\
0,82 b c \\
0,87 b\end{array}$ & $\begin{array}{l}0,89 b \\
0,77 c \\
0,90 b \\
0,78 b c\end{array}$ & $\begin{array}{l}1,29 a \\
1,03 a \\
1,07 a \\
1,08 a\end{array}$ & $\begin{array}{l}0,82 b \\
0,68 d \\
0,72 c \\
0,73 c\end{array}$ \\
\hline
\end{tabular}

(1) SC: sistema convencional, CM: cultivo mínimo, SD: semeadura direta e PG: pré-germinado. ${ }^{(2)}$ Médias seguidas da mesma letra, na linha, não diferem pelo teste de Duncan a $5 \%$.

Quadro 2. Correlações si mples entre diâmetro médio ponderado (DMP) de agregados e C-orgânico e entre DMP e Argila naturalmente dispersa em água (ADA) de um Planossolo, considerando sistemas de manejo e camadas do solo

\begin{tabular}{ccccc}
\hline \multirow{2}{*}{ Variável } & \multicolumn{4}{c}{ Coeficiente de correlação } \\
\cline { 2 - 4 } & $\mathbf{0 - 2 , 5} \mathbf{~ c m}$ & $\mathbf{2 , 5 - 5 , 0 ~ c m}$ & $\mathbf{5 , 0 - 1 0 ~ c m ~}$ & $\mathbf{1 0 - 2 0} \mathbf{~ c m}$ \\
\hline DMP x C-orgânico & $0,782^{* *}$ & $0,453^{* *}$ & $0,549^{* *}$ & $0,632^{* *}$ \\
DMP x ADA & $-0,043 \mathrm{~ns}$ & $0,060 \mathrm{~ns}$ & $0,267 \mathrm{~ns}$ & $0,135 \mathrm{~ns}$ \\
\hline
\end{tabular}

**: Significativo a 1 \% e ns: Não-significativo. 


\section{CONCLUSÕES}

1. O sistema de semeadura direta favoreceu a formação de agregados de maior tamanho, originando maior diâmetro médio ponderado de agregados, enquanto o sistema de plantio de arroz pré-germinado proporcionou a maior concentração de agregados do solo na classe de menor tamanho, com menor diâmetro médio ponderado dos agregados.

2. O diâmetro médio ponderado dos agregados do sol o correlacionou-se, linear e positivamente, com o C-orgânico, não apresentando correlação com a argila naturalmente dispersa em água.

\section{LITE RATURA CITADA}

ARSHAD, M.A.; LOWERY, B. \& GROSSMAN, B. Physical tests for monitoring soil quality. I n: DORAN, J.W. \& J ONES, A.J ., eds. Methods for assessing soil quality. Madison, Soil Science Society of America, 1996. p.123-141.

BAYER, C. \& MIELNICZUK, J. Dinâmica e função da matéria orgânica. In: SANTOS, G. A.\& CAMARGO, F.A.O., eds. Fundamentos da matéria orgânica do solo: ecossistemas tropicais e subtropicais. Porto Alegre, Gênesis, 1999. p.926.

BAYER, C. \& MIELNICZUK, J. Características químicas do solo afetado por métodos de cultura. R. Bras. Ci. Solo, 21:105112, 1997.

BRASIL, Ministério da Agricultura. Levantamento de reconhecimentos dos sol os do Estado do Rio Grande do Sul. Recife, Departamento Nacional de Pesquisa Agropecuária, Divisão de Pesquisa Pedológica. 1973. 431p. (Boletim Técnico, 30)

CARPENEDO, V. \& MIELNICZUK, J. Estado de agregação e qualidade e agregados de Latossolos Roxos, submetidos a diferentes sistemas de manejo. R. Bras. Ci. Solo, 14:1:99105, 1990.

CASTRO FILHO, C.; MUZILLI, O. \& PODANOSCHI, A.L. Estabilidade dos agregados e sua relações com o teor de carbono orgânico num Latossolo Roxo distrófico, em função de sistemas de plantio, rotações de culturas e método de preparo das amostras. R. Bras. Ci. Solo, 22:527-538, 1998.

DORAN, J.W. \& PARKIN, T.B. Defining and assessing soil quality. In: DORAN, J.W.; COLEMAN, D.C.; BEZDICEK, D.F. \& STEWARD, B.A., eds. Defining soil quality for sustainable environment. Madison, Soil Science Society of American, American Society of Agronomy, 1994. p.3-21.

EASH, N.S.; KARLEN, D.L. \& PARKIN,T.B. Fungal contributions to soil aggregation and soil quality. In: DORAN, J.W.; COLEMAN, D.C.; BEZDICEK, D.F. \& STEWART, B.A., eds. Defining soil quality for a sustainable environment. Madison, Soil Science Society of America, American Society of Agronomy, 1994. p.221-228.
ELTZ, F.L.F.; PEIXOTO, R.T.G. \& J ASTER, F. Efeitos de sistema de preparo do solo nas propriedades físicas e químicas de um Latossolo Bruno álico. R. Bras. Ci. Solo, 13:259-267, 1989.

EMPRESA BRASILEIRA DE PESQUISA AGROPECUÁRIA EMBRAPA. Centro Nacional de Pesquisa de Solos. Sistema Brasileiro de Classificação de solos. Brasília, 1999. 412p.

GEE, G.W. \& BAUDER, J.W. Particle size analysis. In: KLUTE, A., ed. Methods of soil analysis. 2.ed. Madison, American Society of Agronomy, Soil Science Society of America, 1986. p.383-411.

INSTITUTO RIOGRANDENSE DO ARROZ -IRGA. Arroz Irrigado: Recomendações técnicas de pesquisa para o sul do Brasil. IRGA. Porto Alegre, 2001. 128p.

KEMPER, W.D. \& ROSENAU, R.C. Aggregate stability and size distribution. In: KLUTE, A., ed. Methods of soil analysis. 2.ed. Madison, American Society of Agronomy, Soil Science Society of America, 1986. p.425-441.

PALMEIRA, P.R.T.; PAULETTO, E.A.; TEIXEIRA, C.F.A.; GOMES, A.S. \& SILVA, J.B. Agregação de um Planossolo submetido a diferentes sistemas de cultivo. R. Bras. Ci. Solo, 23:189-195, 1999.

PEÑA, Y.A.; GOMES, A.S. \& SOUSA R.O. Influência de diferentes sistemas de cultivo nas propriedades físicas de um solo de várzea cultivado com arroz irrigado. R. Bras. Ci. Solo, 20:517-523, 1996.

REICHERT, J.M.; VEIGA, M. \& CABEDA, M.S.V. Índices de estabilidade de agregados e suas relações com características e parâmetros. R. Bras. Ci. Solo, 17:283-290, 1993.

SILVA, I.F. \& MIELNICZUK, J . Avaliação do estado de agregação do solo afetado pelo uso agrícola. R. Bras. Ci. Solo, 21:313319, 1997a.

SILVA, I.F. \& MIELNICZUK J. Ação do sistema radicular de plantas na formação e estabilização de agregados do solo. R. Bras. Ci. Solo, 21:113-117, 1997b.

SILVA I.F. \& MIELNICZUK J. Sistemas de cultivo e características do sol o afetado a estabilidade de agregados. R. Bras. Ci. Solo, 22:311-317, 1998.

SILVA, L.S.; CAMARGO, F.A.O. \& CERETTA C.A. Composição da fase sólida do solo. In: MEURER E.J ., ed. Fundamentos da química do solo. Porto Alegre, Gênesis, 2000a p.45-62.

SILVA, V.R.; REINERT, D.J . \& REICHERT, J.M. Densidade do solo, atributos químicos esistema radicular do milho afetado pelo pastejo e manejo do solo. R. Bras. Ci. Solo, 24:191-199, 2000b.

SOIL SURVEY STAFF. Keys to soil taxonomy. 4.ed. Virgínia, 1990. 422p. (SMSS technical monograph, 6)

TEDESCO, M.J .; GIANELLO, G.; BISSANI, C.A.; BOHNEN, H. \& VOLKWEISS, S.J. Análises de solo, plantas e outros materiais. 2.ed. Porto Alegre, Universidade Federal do Rio Grande do Sul, 1995. 174p. 
TEIXEIRA C.F.A. Influência de sistemas de cultivo em plantio direto em alguns atributos físicos de um Podzólico Vermelho-Amarelo. Pelotas, Universidade Federal de Pelotas, 1998. 49p. (Tese de Mestrado)

TISDALL, J .M. \& OADES, J .M. Organic matter and water stable aggregates in soil. J. Soil Sci. 33:141-163, 1982.
YODER, R.E. A direct method of aggregate analysis of soils and a study of the physical nature of erosion losses. J. Am. Soc. Agron., 28:337-351, 1936.

ZONTA, E.P.; MACHADO, A.A. \& SILVEIRA, J.P. Sistema de análise estatística para microcomputadores - SANEST. Pelotas, Universidade Federal de Pelotas, 1984. 151p. 